\title{
Percepción sobre el desempeño docente y gestión del conocimiento en estudiantes de educación secundaria
}

\author{
Perception on teaching performance and knowledge \\ management in high school students
}

\author{
Eugenia Yolanda Alván More \\ Universidad Nacional de Educación Enrique Guzmán y Valle
}

\section{Resumen}

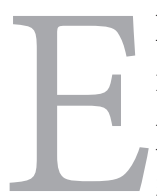

1 objetivo principal de la investigación fue establecer el grado de relación entre percepción del desempeño docente y gestión de conocimientos en estudiantes de educación secundaria. El diseño de estudio es correlacional y la muestra estuvo conformada por 86 estudiantes a quienes se les administró dos cuestionarios el de percepción de desempeño docente, que se elaboró en base al Manual de buenas prácticas de evaluación del desempeño profesional de los docentes de Valdez (2009), y el de gestión del conocimiento, que se elaboró en base a la obra, autoevaluación de la calidad educativa en escuelas secundarias de UNICEF-CEADEL (2013). A partir del análisis de los datos obtenidos se pudo demostrar que existe una relación significativa entre desempeño docente y gestión del conocimiento de los estudiantes.

\section{Palabras clave:}

Desempeño docente, gestión del conocimiento.

\section{Summary}

he objective of the present study was to determine the existing relationship between teaching performance and knowledge management in students from the first and second year of high school. It has a correlational design. the sample was composed by 86 high school students, who were administered two questionnaires; the one for teaching performance, which was base on the "Manual de Buenas Prácticas de Evaluación del Desempeño Profesional de los Docentes" from Valdez (2009), and the one for the knowledge management, which was based on the work of "Self-evaluation of the Education Quality in High Schools" from UNICEF-CEADEL (2013). From the result and the analysis it is can demonstrate that there is a significant relationship between teaching performance and knowledge management in high school students.

\section{Key words:}

Teaching performance and knowledge management. 


\section{Ciencia}

\section{Introducción}

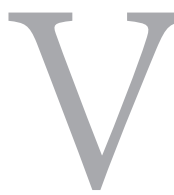

ivimos a un ritmo muy acelerado en los cambios, tanto en lo que se refiere a la tecnología como a la información

y el conocimiento. Sabemos que a través de la información surge el conocimiento, una vez que aquella ha dado significado a los datos, con el apoyo inexcusable de la educación, de las experiencias previas y de los valores asumidos, podemos comprender todas las representaciones contextuales. El conocimiento se produce por una confluencia, entre otros, de dos factores sustanciales, la información indicada (búsqueda, procesamiento, almacenamiento) y la comunicación (distribución e intercambio de información). Esos procesos se activan de forma sistemática a través de la educación, para el cual, se hace preciso el uso de recursos, herramientas, aplicaciones, redes y entornos tecnológicos que configuran elementos esenciales de esta sociedad (García, 2012).

En este contexto, el docente del siglo XXI tiene que estar en la vanguardia de los nuevos conocimientos, las nuevas estrategias a aplicar a los estudiantes y sobre todo tener una actitud de disposición hacia los nuevos contextos de aprendizaje y enseñanza. En esto consiste el buen desempeño docente entendido como "el cumplimiento de sus funciones los cuales son factores asociados al docente, al estudiante y al entorno. Asimismo se ejerce en diferentes campos o niveles: en el contexto socio-cultural, entorno institucional, ambiente de aula y sobre todo en el propio dicente, mediante una acción reflexiva" (Delanoy 2001, p.201).

Por ello, las organizaciones educativas constituyen ámbitos específicos de generación, gestión y transmisión del conocimiento, que afrontan nuevas demandas provenientes de la compleja sociedad del conocimiento, entre ellas las fuertes demandas por la inclusión y la calidad (Tedesco, 2000). Estos constructos estarían entonces relacionados con la percepción del desempeño docente y la gestión del conocimiento por parte de los estudiantes de la Institución Educativa No 1263 Puruchuco de Ate -Vitarte.

El objetivo de esta investigación es determinar si existe relación entre el desempeño de los docentes y la gestión del conocimiento en los estudiantes, teniendo en cuenta tanto el desempeño de los docentes como la habilidad para manejar la información, pueden contribuir a buenos resultados en su aprendizaje y por ende, mejora en la gestión del conocimiento de los educandos apoyados en el trabajo colaborativo.

\section{Metodología}

Participantes. En ese estudio participaron un total de 86 estudiantes de ambos sexos del primer y segundo año de educación secundaria de la institución educativa No 1263 Puruchuco.

Materiales. Se utilizó un cuestionario de percepción de desempeño docente, la cual fue elaborado en base al manual de buenas prácticas de evaluación del desempeño profesional de los 


\section{Ciencia}

docentes de Valdez (2009), para el cual se ha considerado las dimensiones afectiva $(1,2,3,4)$ y didáctica $(5,6,7,8)$. Y para la evaluación de gestión del conocimiento, se realizó un cuestionario basado en la autoevaluación de la calidad educativa en escuelas secundarias de UNICEF-CEADEL (2013), para el cual se ha considerado las dimensiones que corresponden: contexto cognitivo ( 9 , $10,11,12)$; transferencia de conocimiento $(13,14$, $15,16,17,18,19)$ y aprendizaje organizacional $(20,21,22,23,24)$.
La validez de los instrumentos según Alfa de Cronbach proporciona un puntaje, para desempeño docente 0.693 y para gestión del conocimiento es de 0.883 con lo cual los instrumentos son aceptables.

\section{Resultados}

Se analizan los resultados en función al análisis de los datos estadísticos, a partir del cual se evidencia que:

Tabla 1.

Prueba para una muestra

\begin{tabular}{lcccccc} 
& \multicolumn{6}{c}{ Valor de prueba $=22$} \\
\cline { 2 - 7 } $\begin{array}{l}\text { Percepción } \\
\text { del desempeño } \\
\text { docente }\end{array}$ & $\mathrm{t}$ & $\mathrm{gl}$ & $\begin{array}{c}\text { Sig. } \\
\text { (bilateral) }\end{array}$ & $\begin{array}{c}\text { Diferencia } \\
\text { de medias }\end{array}$ & $\begin{array}{c}\text { 95\% Intervalo de confianza } \\
\text { para la diferencia }\end{array}$ \\
\cline { 2 - 7 } & 5.210 & 85 & .000 & 2.163 & Inferior & Superior \\
\hline
\end{tabular}

De la tabla anterior se sabe que $t_{C}=5.210$

Se puede inferir con un nivel de significación de 0.05 que, los estudiantes de la Institución Educativa No 1263 Puruchuco de Ate -Vitarte, pre-

Tabla 2.

Prueba para una muestra

\begin{tabular}{|c|c|c|c|c|c|c|}
\hline \multicolumn{7}{|c|}{$\begin{array}{l}\text { Tabla } 2 . \\
\text { Prueba para una muestra }\end{array}$} \\
\hline \multirow{4}{*}{$\begin{array}{l}\text { Gestión del } \\
\text { conocimiento }\end{array}$} & \multicolumn{6}{|c|}{ Valor de prueba $=32$} \\
\hline & $\mathrm{t}$ & $\mathrm{gl}$ & $\begin{array}{c}\text { Sig. } \\
\text { (bilateral) }\end{array}$ & $\begin{array}{l}\text { Diferencia } \\
\text { de medias }\end{array}$ & \multicolumn{2}{|c|}{$\begin{array}{l}\text { 95\% Intervalo de confianza } \\
\text { para la diferencia }\end{array}$} \\
\hline & \multirow{2}{*}{13.259} & \multirow{2}{*}{85} & \multirow{2}{*}{.000} & \multirow{2}{*}{11.267} & Inferior & Superior \\
\hline & & & & & 9.58 & 12.96 \\
\hline
\end{tabular}

De la tabla anterior se sabe que $t_{C}=13.259$ sentan un nivel alto de percepción del desempeño de sus docentes. 


\section{Ciencia}

Se puede inferir con un nivel de significación de 0.05 que, existe un nivel alto de gestión de conocimiento en estudiantes de la Institución Educativa No 1263 Puruchuco de Ate -Vitarte.

Se quiere determinar la relación entre la percepción del desempeño docente y gestión de conocimiento en estudiantes de la Institución Educativa No 1263. Para ello analizaremos el tipo de distribución que presentan los datos en cada variable, a través de la prueba de kolmogorov.
Considerando el valor de la prueba, se determinara el uso del estadístico de correlación: si los datos presentan distribución normal, se utilizara el paramétrico (Pearson), caso contrario, no paramétrico (Rho de Spearman).

Prueba de normalidad: Kolmogorov. Es una prueba de bondad de ajuste, permite determinar si los datos provienen de una población con distribución normal.

Tabla 3

Prueba de Kolmogorov-Smirnov para una muestra

\begin{tabular}{llcc} 
& & \multicolumn{1}{c}{$\begin{array}{c}\text { Percepción } \\
\text { desempeño docente }\end{array}$} & $\begin{array}{c}\text { Gestión del } \\
\text { conocimiento }\end{array}$ \\
\hline $\mathrm{N}$ & & 86 & 86 \\
\hline \multirow{2}{*}{ Parámetros normales $(\mathrm{a}, \mathrm{b})$} & Media & 24.16 & 43.27 \\
\cline { 2 - 4 } & Desviación típica & 3.850 & 7.881 \\
\hline \multirow{2}{*}{ Diferencias más extrema } & Absoluta & .133 & .068 \\
\cline { 2 - 4 } & Positiva & .070 & .068 \\
\cline { 2 - 4 } & Negativa & -.133 & .046 \\
\hline Z de Kolmogorov-Smirnov & & 1.230 & .628 \\
\hline Sig. asintót. (bilateral) & & .097 & .825 \\
\hline a La distribución de contraste es la Normal. \\
b Se han calculado a partir de los datos.
\end{tabular}

De acuerdo a los resultados obtenidos en la (sig bilateral), dicho resultado es mayor a 0.05, por prueba de kolmogorow para la variable percepción del desempeño docente, el valor de $\mathrm{p}=0.097$ 


\section{Ciencia}

\begin{tabular}{|c|c|c|c|c|c|}
\hline \multicolumn{6}{|c|}{$\begin{array}{c}\text { Tabla } 4 \\
\text { Correlación de variables }\end{array}$} \\
\hline & & $\begin{array}{l}\text { Gestión del } \\
\text { conocimiento }\end{array}$ & $\begin{array}{l}\text { Contexto } \\
\text { cognitivo }\end{array}$ & $\begin{array}{l}\text { Transferencia de } \\
\text { conocimientos }\end{array}$ & $\begin{array}{c}\text { Aprendizaje } \\
\text { organizacional }\end{array}$ \\
\hline \multirow{3}{*}{$\begin{array}{l}\text { Percepción del } \\
\text { desempeño } \\
\text { docente }\end{array}$} & $\begin{array}{l}\text { Correlación de } \\
\text { Pearson }\end{array}$ & $.482\left(^{* *}\right)$ & & & \\
\hline & Sig.(bilateral) & 000 & & & \\
\hline & $\mathrm{N}$ & 86 & & & \\
\hline factor: afectiva & & & $\left..4999^{(* *}\right)$ & $\left..3333^{(* *}\right)$ & $.4300^{* *}$ \\
\hline Factor: didáctica & & & $.263\left(^{*}\right)$ & $\left..2888^{(* *}\right)$ & $\left..405^{(* *}\right)$ \\
\hline
\end{tabular}

Entonces existe una relación estadísticamente significativa entre la percepción del desempeño docente y gestión de conocimiento a nivel general; y en la relación entre las dimensiones de cada variable, se evidencia que también existe relación significativa en cada una de ellas, de esta manera se afirma que entre estas variables según Hernández, Fernández y Baptista (2014) existe una relación positiva media o moderada.

\section{Discusión}

A nivel descriptivo los estudiantes tienen una percepción alta del desempeño docente. Estos resultados se debería a que los estudiantes perciben una eficiencia, un accionar estratégico de sus maestros para llegar mejor a ellos, orientación metodológica y lo que se refiere a la toma de decisiones en el quehacer educativo, además, concuerdan con las propuesta de Fernández (2010), Valdés (2004) y Montenegro (2003) que coinci- den en señalar que existen una cierta cantidad de acciones del docente que influye para que los estudiantes puedan mejorar, sobre todo en la motivación de aprendizaje. Por otro lado, un nivel alto de gestión de conocimiento en los resultados, no son tan alarmantes en estos tiempos, debido a que los estudiantes perciben que sus maestros están ingresando principalmente al reino virtual en el que se mueve la sociedad del conocimiento y la información. Sin embargo, un porcentaje menor de estudiantes perciben que aún existe una gran cantidad de maestros que está intentando ingresar y pero que aún existe una serie de retos y posibilidades para que puedan aprender, desde cómo usar las nuevas tecnologías en el proceso de gestionar el conocimiento, innovar programas, integrar un mayor número de estudiantes hasta como adecuar y modificar las estructuras organizacionales de las instituciones educativas para acoger y liderar la educación (Hidalgo, 2011). 


\section{Ciencia}

A nivel inferencial, es notable observar que existe una correlación positiva media o moderada, entre la percepción del desempeño docente y gestión de conocimiento en la muestra estudiada. Estos resultados parecen un poco preocupante en estos tiempos, donde la disponibilidad de información y la posibilidad de dominar una tecnología es infinita. Además, los estudiantes perciben que los docentes aun no aplican en sus quehacer educativos algunos principios fundamentales que para Nonaka y Takeuche (1995, citado por Minakato, 2009) se realiza en cuatro fases: la de socialización, de compartir experiencias e ideas personales del conocimiento tácito personal al conocimiento colectivo; la de externalización, la del paso del conocimiento tácito colectivo al explícito; la de combinación, en la que se efectúa el intercambio de conocimientos explícitos, documentos compartidos por diferentes modalidades, principalmente electrónicas y virtuales; y la de interiorización o de aprendizaje, en la que el conocimiento explícito colectivo se transforma en tácito individual.

En cuanto a los factores de cada variable existen correlaciones positiva media o moderada. Estos resultados necesariamente tendrían que ver con uno de los componentes principales de la gestión del conocimiento, debido a que es el punto central de este proceso, tanto para los docentes como para los estudiantes, dado que en ello, se configuran los constitutivos de la gestión del conocimiento: procesos y ciclos, mediaciones instrumentales y componentes de aprendizaje grupal y "organizacional", elementos que son los ejes de la gestión del conocimiento y se visualizan como una movilización, el hacer que las cosas sucedan en el ciclo de producción (generación) - intermediación - aplicación del conocimiento con el apoyo de tecnologías de información y comunicación (Hidalgo, 2011). Estos componentes centrales son la creación, circulación y transferencia de conocimiento en una institución y contexto en particular, es decir, es la organización entre los actores y las acciones que deben darse. 


\section{Ciencia}

\section{Referencias}

Delannoy, F (2001). Profesionalismo docente y aseguramientodelacalidaddelaenseñanza.Ponencia presentadaen el Seminario InternacionalProfesionalismodocentey Calidad de la Educación. Santiago. Recuperado de http://www.mineduc.cl/zonas/ profesores/seminario

Fernández,E. (2010). Predictores emocionalesy cognitivosdelbajorendimientoacadémicounenfoquebiopsicoeducativo.Málaga: Universidad de Málaga.

García,L(2012).Sociedaddelconocimientoyeducación. Madrid: Aranzadi.

Hidalgo, L. (2011). El docente y la gestión del conocimiento en la educación superior a distancia. Recuperado el $1^{\circ}$ de abril de 2015. Recuperado de http://reposital.cuaed.unam.mx:8080/jspui/bitstream/123456789/2740/1/lilliam_hidalgo _docente_gestion_del_conocimiento.pdf

Minakata, A. (2009). Gestión del conocimiento en educación y transformación de la escuela. Notas para un campo en construcción. Sinéctica, 32,1-35. Recupera- do de http://www.redalyc.org/articulo. oa?id=99812141008

Montenegro,A.(2003). EvaluacióndelDesempeño Docente. Bogotá: Magisterio.

Nonaka, I. y Takeuchi, H. (1995). The KnowledgeCreatingCompany.HowJapaneseCompanies CreatetheDynamics of Innovation. Oxford, UK: Oxford University Press

Tedesco,J.(2000).Educarenlasociedaddelconocimiento. Buenos Aires: Fondo de Cultura Económica

UNICEF-CEADEL (2013). Autoevaluación dela calidadeducativaenescuelassecundarias. UncaminoparamejorarlaCalidadEducativaenescuelassecundarias.BuenosAires: Alicia de Santos y Alejandra Piatigorsky.

Valdés,H. (2004). Eldesempeñodelmaestroysu evaluación. Habana: Pueblo y educación.

Valdés, H.(2009).Manualdebuenas prácticas de evaluacióndeldesempeñoprofessionalde los docentes. Lima: Tarea Asociación Gráfica Educativa. 